\title{
Self-generation of self-replicating maps of an interval
}

\author{
WILLIAM PARRY $\dagger$ \\ From the Mathematics Institute, University of Warwick, England
}

(Received 6 November 1980)

\begin{abstract}
We construct $C^{1}$ symmetric maps of $[-1,1]$ to itself, satisfying a condition similar to Feigenbaum's: $-\phi(x)=\phi(-x), \phi^{2}(b x)=b \phi(x), 0<b<1$. Under certain conditions, the non-wandering set consists of the one-sided Morse minimal set together with $2^{n}$ points of period $2^{n}$ for each $n=1,2, \ldots$ The main significance of the construction is its simplicity. Given a certain piece of the map $\phi$, the rest is generated by the required equation.
\end{abstract}

\section{Introduction}

Families of maps of an interval have received considerable attention recently, especially with regard to bifurcation phenomena. A remarkable bifurcation picture emerges in [4] and [10]; the first concerns the support of the natural invariant measure and the second concerns spectral and Bernoulli properties for the twodimensional family of maps $x \rightarrow \beta x+\alpha \bmod 1$. In [11] we investigated a onedimensional sub-family of these maps, namely, the centrally symmetric ones. (A related study appears in [8].)

If $\alpha$ is chosen so that $T_{\beta} x=\beta x+\alpha \bmod 1$ is symmetric about $\frac{1}{2}(\alpha=1-\beta / 2)$ then, as $\beta$ decreases from $\sqrt{ } 2$ to 1 , periodic intervals and associated periodic points are created with larger and larger (doubling) periods. In the range $2^{1 / 2^{n+1}}<\beta \leq 2^{1 / 2^{n}}$ $(n=1,2, \ldots)$ there is an interval $I$ such that $T_{\beta}^{2} I=I\left(T_{\beta} I=J \cup J^{\prime}\right.$ where $I, J, J^{\prime}$ are pairwise disjoint) and $T_{\beta}^{2} \mid I$ is linearly isomorphic to $T_{\beta^{2}}$. Thus the family $\left\{T_{\beta}: 1<\beta<\sqrt{2}\right\}$ is re-normalizing in the jargon of physicists.

The question arises as to whether this phenomenon can occur with a single map, and if so what properties would such a map enjoy?

This question has been studied extensively in [2] and [1], the former by computer experiment and the latter rigorously and globally, for maps with a single (maximum) critical point. The maps we shall concentrate on retain the general shape of the maps $T_{\beta}$ in that they are maps associated with a Lorenz semi-flow (cf. [3], [12]). We shall therefore call them Lorenz type maps since they occur as the Poincaré section maps of a Lorenz semi-flow.

$\dagger$ Address for correspondence: Professor William Parry, Mathematics Institute, University of Warwick, Coventry CV4 7AL, England. 


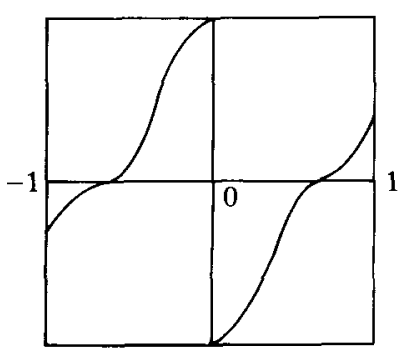

Figure 1. Lorenz type.

We shall construct maps $\phi$ of $[-1,1]$ to itself which are centrally symmetric $(\phi(-x)=-\phi(x))$ satisfying

$$
\phi^{2}(b x)=b \phi(x) \quad \text { where } 0<b<1 .
$$

$\phi$ will be slightly improper in that $\phi$ has two values at 0 , namely $\phi(0)= \pm 1$. Otherwise $\phi$ will be strictly increasing and continuous, with a graph such as figure 1 . Figure 2 represents the map $\psi$ obtained by factoring the $\mathbb{Z}_{2}$ symmetry $x \rightarrow-x$. Thus $\psi$ will satisfy the equation

$$
\psi^{2}(b x)=b \psi(x), \quad(\psi(x)=|\phi(x)|)
$$

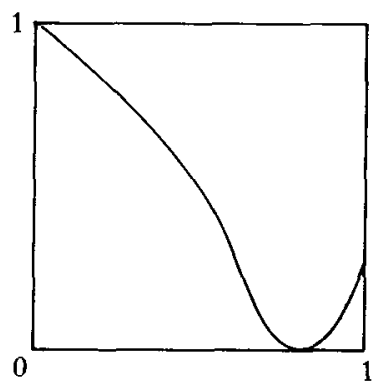

Figure 2

Feigenbaum introduced the equation $\phi^{2}(b x)=-b \phi(x)$ appropriate to his class of maps. The advantage of studying the Lorenz type maps is that they can be constructed; indeed, if we prescribe a certain part of the map, the remainder is self-generated by the equation $(0.1)$.

In this paper there is no pretension to generality. The novelty and interest of our results lie in the following:

(a) The maps we construct are self-generating.

(b) Conditions are given for the non-wandering set to consist of $2^{n}$ periodic points of period $2^{n}$ for $n=1,2, \ldots$ together with the limit set of these periodic points which is, essentially, the Morse minimal set.

We conclude with some remarks concerning the map $\psi$ of the unit interval and with computations of the characteristic exponents of the map $\phi$ (and $\psi$ ).

For further literature to be consulted which relates closely to this paper cf. [9], $[\mathbf{8}],[5]$. 
I would like to acknowledge several interesting conversations with Bob Williams and David Rand concerning my constructions.

The reader may find the following quotation from Warning to Children by Robert Graves, a suitable preparation for the phenomena discussed in this paper:

Children leave the string untied!

For who dares undo the parcel

Finds himself at once inside it,

On the island, in the fruit,

Blocks of slate about his head,

Finds himself enclosed by dappled

Green and red, enclosed by yellow

Tawny nets, enclosed by black

And white acres of dominoes,

But the same brown paper parcel

Still untied upon his knee.

And, if he then should dare to think

Of the fewness, muchness, rareness,

Greatness of this endless only

Precious world in which he says

He lives - he then unties the string.

\section{Geometric behaviour}

Before constructing $\phi$ let us look at some of the properties of maps (see figure 1) satisfying $\phi^{2}(b x)=b \phi(x)$.

The Lorentz semi-flow is pictured in figure 3 . Thus $\phi$ maps $[-b, 0]$ to $[a, 1]$ and $[0, b]$ to $[-1,-a]$. Between $-a$ and $-b$ and between $b$ and $a$ are two points of period 2 (figure $4(a)$ ).

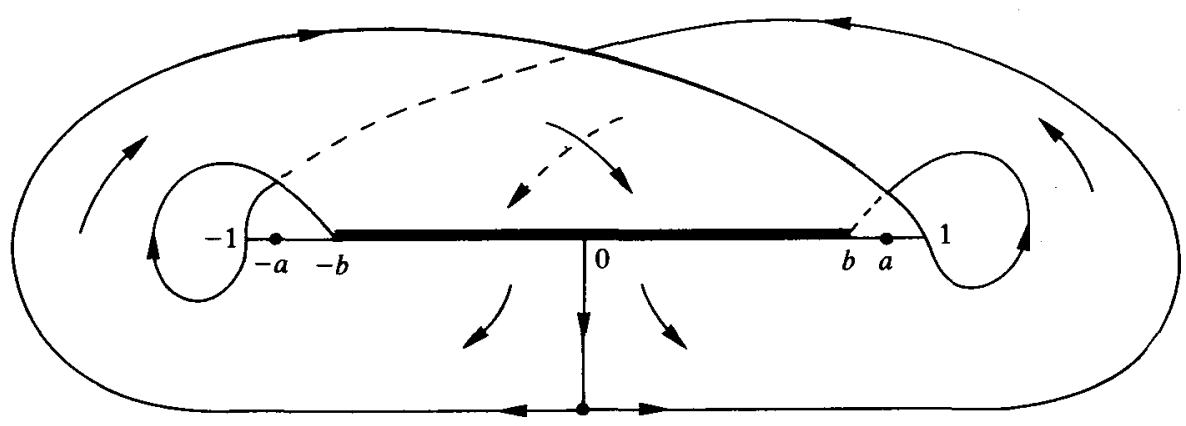

FIGURE 3. Lorenz semi-flow. Phase space a branched surface with branch line $[-b, b]$. The point 0 moves toward a saddle point singularity, $-b$ moves clockwise and over the picture to meet $[-1,1]$ in $a . b$ moves anti-clockwise and behind the picture to meet $[-1,1]$ in $-a$.

Such pictures can be repeated ad infinitum (figure $4(b)$ ). Except for the two periodic points, $(-a,-b) \cup(b, a)$ consists of wandering points. The self-replicating behaviour of $\phi$ is further illustrated by the period doubling pictured in figure 5 . 


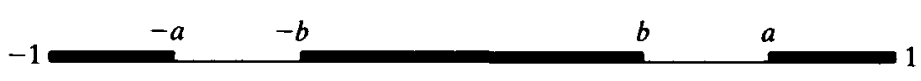

FIGURE $4(a)$. The interval $[-b, b]$ together with its $\phi$ image.

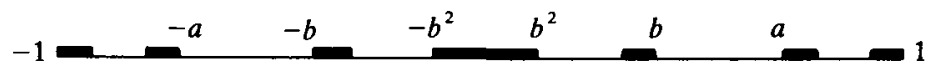

FIGURE $4(b)$. The behaviour of $\phi$ described in figure $4(a)$ is copied in $[-b, b]$ with respect to $\phi^{2}$.

\section{The construction}

Let $\phi$ be a map defined on $[-1,-d] \cup[-e, e] \cup[d, 1]$ with range a subset of $[-1,1]$, where $0<e \leq d \leq 1$, satisfying:

(i) $\phi$ is single-valued everywhere except at $0(\phi(0)= \pm 1)$.

(ii) $\phi$ is strictly increasing and continuous on $[-1,-d] \cup[-e, 0]$ (choosing $\phi(0)=$ 1) with range a subset of $[-b, 1]$.

(iii) $\phi(x)=-\phi(-x)$.

Such a map will be called partially Lorenz.

If $\phi$ satisfies

(iv) $\phi(x)=(1 / b) \phi^{2}(b x)$ (where $\left.0<b<1\right)$, when both sides are defined, then $\phi$ is called self-replicating.

If $\phi$ is defined on the entire interval $[-1,1]$ and satisfies (i), (ii), (iii), then $\phi$ is called a Lorenz map. Our aim is to construct self-replicating Lorenz maps.

Let $0<b<a<1$ and let $\phi(-1)=-b, \phi(1)=b, \phi(-b)=a, \phi(b)=-a, \phi(-a)=$ $b^{2}, \phi(a)=-b^{2}$. Moreover, let $\phi$ be a strictly increasing continuous function defined on $[-a, 0]$ with $\phi(0)=1$. Define $\phi$ on $[0, a]$ by $\phi(x)=-\phi(-x)$. Then $\phi$ is a self-replicating partial Lorenz map with domain $\{-1\} \cup\{1\} \cup[-a, a]$. (iv) is satisfied since $x= \pm 1$ are the only points where both sides of this equation are defined and the equation is satisfied for these points.

If $x \in[-a, 0]$ then $\phi(x)$ is well defined and $\phi(b x) \in[\phi(-b a), 1]$. (Note that $a=\phi(-b)<\phi(-b a)$.) Hence we can define $\phi(y)=b \phi(x)$ for $y=\phi(b x), x \in[-a, 0]$ so that (iv) is satisfied. (If $x=0$, then $y=1$ and $\phi(1)=b$, which agrees with the way we defined $\phi$ initially.)

Similarly, or by symmetry, we can define $\phi$ on $[-1, \phi(b a)]$. We now have $\phi$ defined on

and

$$
\begin{gathered}
{[-1, \phi(b a)] \cup[-a, a] \cup[\phi(-b a), 1]} \\
-b^{2}=\phi(a)<\phi^{2}(-b a)=b \phi(-a)=b^{3}
\end{gathered}
$$

so that $\phi$ continues to satisfy (i), (ii), (iii).

Let the map $x \rightarrow b x$ also be denoted by $b$ then $\phi(-b a)=(\phi b)^{2}(1)$ and the domain of $\phi$ is

$$
\left.\left[-1,(\phi b)^{2}(-1)\right] \cup[\phi b(1), \phi b(-1)] \cup[\phi b)^{2}(1), 1\right]
$$

Suppose we have extended $\phi$ to a self-replicating partially Lorenz map with domain

$$
\left[-1,(\phi b)^{2 n}(-1)\right] \cup\left[(\phi b)^{2 n-1}(1),(\phi b)^{2 n-1}(-1)\right] \cup\left[(\phi b)^{2 n}(1), 1\right]
$$

where these three intervals are pairwise disjoint. 


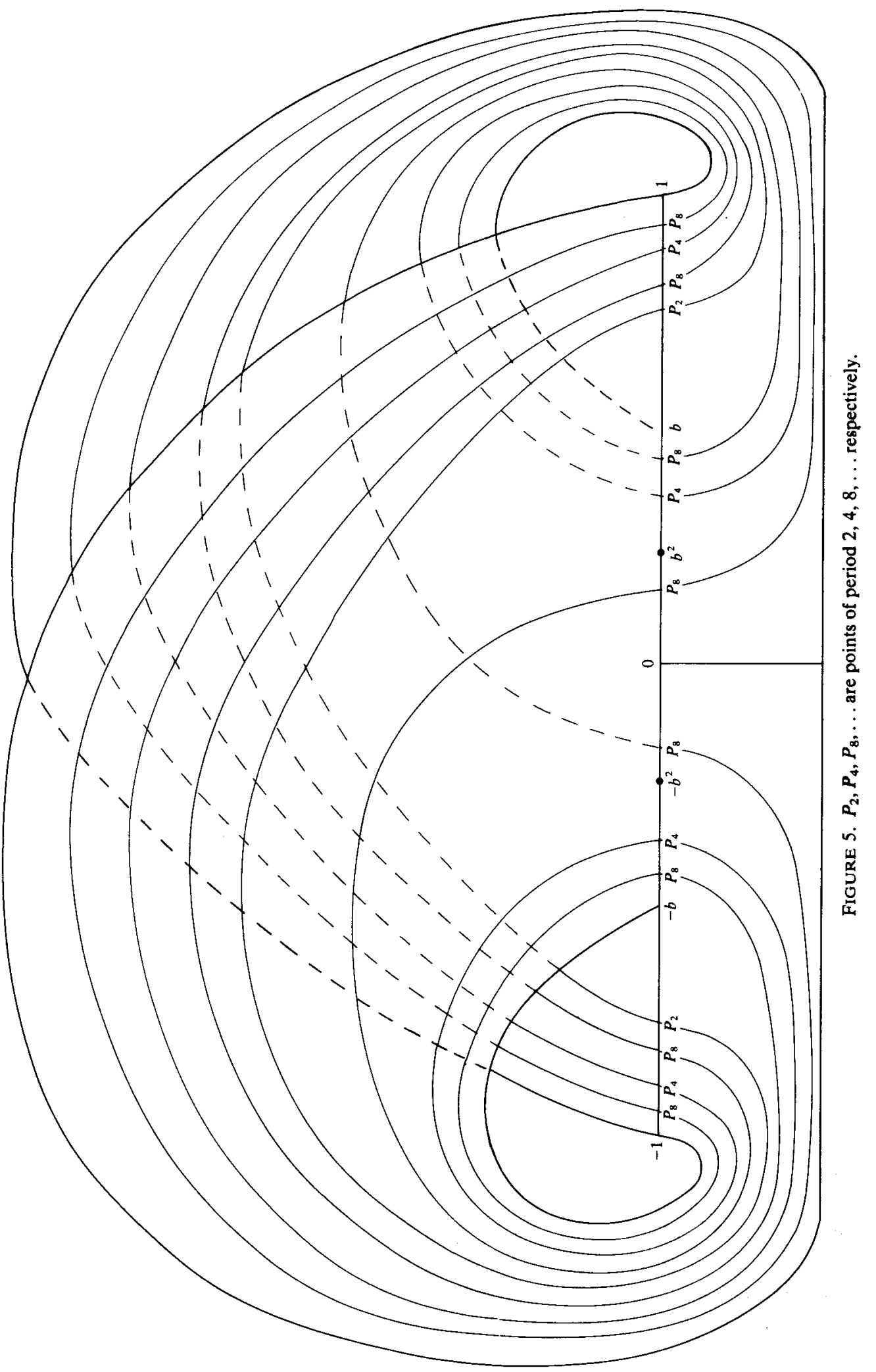


If $x \in\left[(\phi b)^{2(n-1)}(-1),(\phi b)^{2 n}(-1)\right]$ then $\phi b x \in\left[(\phi b)^{2 n-1}(-1),(\phi b)^{2 n+1}(-1)\right]$ and we define $\phi(\phi b x)=b \phi(x)$ and note that $\phi$ so defined assigns the value

$$
b \phi\left((\phi b)^{2(n-1)}(-1)\right)=b^{2} \phi(\phi b)^{2 n-3}(-1)=\cdots=b^{2 n-1}(-1) \quad \text { to }(\phi b)^{2 n-1}(1),
$$

which is the correct value for consistency.

Again $\phi$ can be defined on $\left[(\phi b)^{2 n+1}(1),(\phi b)^{2 n-1}(1)\right]$ by symmetry.

We should also note that $\phi\left((\phi b)^{2 n}(-1)\right)<\phi\left((\phi b)^{2 n+1}(1)\right)$ since $-b^{2 n}<b^{2 n+1}$. Hence $\phi$ has been extended to a self-replicating partially Lorenz map defined on

$$
\left[-1,(\phi b)^{2 n}(-1)\right] \cup\left[(\phi b)^{2 n+1}(1),(\phi b)^{2 n+1}(-1)\right] \cup\left[(\phi b)^{2 n}(1), 1\right] .
$$

Note that $(\phi b)^{2 n}(1)$ and $(\phi b)^{2 n+1}(-1)$ are decreasing and increasing sequences, respectively.

Let $\alpha=\lim _{n \rightarrow \infty}(\phi b)^{2 n}(1)$ and $\beta=\lim _{n \rightarrow \infty}(\phi b)^{2 n+1}(-1),(\beta \leq \alpha)$, then we can define $\phi$ on

$$
[-1,-\alpha] \cup[-\beta, \beta] \cup[\alpha, 1]
$$

by continuity, noting that $\phi(\alpha)=\phi(\beta)=0\left(=\lim _{n \rightarrow \infty} b^{n}\right)$.

By the definition of $\alpha, \beta$ we have $\phi b(\alpha)=-\beta, \phi b(\beta)=-\alpha$.

At this point we make the following hypothesis:

The initial map $\phi$ has the property that $(\phi b)^{2}$ has no fixed point in $[-1,-a]$.

This is satisfied in particular if

$$
\frac{\phi(x)-\phi(y)}{x-y}<\frac{1}{b} \text { when }-b<y<x<-b a,
$$

for then

$$
\frac{(\phi b)(x)-(\phi b) y}{x-y}<1 \text { when }-1<y<x<-a .
$$

Assuming (H.1) it is clear that $\alpha=\beta$ for otherwise $(\phi b)^{2}$ would have a fixed point. We have proved:

THEOREM 1. Let $0<b<a<1$ and let $\phi$ be a map defined on $[-a, a]$ satisfying (H.1) such that $\phi$ is single-valued everywhere except at $0(\phi(0)= \pm 1), \phi(-a)=b^{2}, \phi(-b)=$ $+a, \phi$ is strictly increasing and continuous in $[-a, 0]$ (taking $\phi(0)=1), \phi(-x)=-\phi(x)$ for all $x \in[-a, a]$, then $\phi$ can be uniquely extended to a self-replicating Lorenz map on $[-1,1]$.

\section{3. $C^{1}$ conditions}

Now let $\phi$ be $C^{1}$ on $[-a, 0], \phi(-b)=a, \phi(0)=1, \phi(-a)=b^{2}, \phi$ strictly increasing and define $\phi(x)=-\phi(-x)$ for $x \in[0, a]$.

THEOREM 2. If the initial map $\phi$ satisfies $1 / b>\phi^{\prime}(x)>1$ in $(-b, 0), \phi^{\prime}(x) \neq 0$ in $(-a, 0)$ and $\phi^{\prime}(-a) \phi^{\prime}(-b)=1$, then the extended map $\phi(c f$. theorem 1$)$ is $C^{1}$ in $[-1,0]$ and in $[0,1]$. 
Proof. Since $\phi(x)=b \phi\left((1 / b) \phi^{-1}(x)\right)$ in a left neighbourhood of 1 , we have

$$
\phi^{\prime}(1)=\phi^{\prime}\left(\frac{1}{b} \phi^{-1}(1)\right) / \phi^{\prime}\left(\phi^{-1}(1)\right)=\phi^{\prime}(0) / \phi^{\prime}(0)=1
$$

and, similarly, $\phi^{\prime}(-1)=1$.

To prove that $\phi$ is $C^{1}$ evidently we have to ensure the continuity of derivatives at 'junctions' $(\phi b)^{n}(1),(\phi b)^{n}(-1)$ and at the limits $\alpha, \beta$. This means that we need $\phi^{\prime}(x)=\phi^{\prime}\left((1 / b) \phi^{-1}(x)\right) / \phi^{\prime}\left(\phi^{-1} x\right)$ at these junctions. In particular, we need

$$
\phi^{\prime}(-a)=\phi^{\prime}(\phi b 1)=\phi^{\prime}\left(\frac{1}{b} \cdot b\right) \phi^{\prime}(b)=1 / \phi^{\prime}(-b)
$$

which is one of our hypotheses.

For $x$ between 1 and $\phi(b)$

$$
\phi^{\prime}\left((\phi b)^{n}(x)\right)=\frac{\phi^{\prime}\left((\phi b)^{n-1}(x)\right)}{\phi^{\prime}\left(b(\phi b)^{n-1}(x)\right)}=\cdots=\frac{\phi^{\prime}(x)}{\phi^{\prime}\left(b(\phi b)^{n-1}(x)\right) \ldots \phi^{\prime}(b x)},
$$

so that

$$
\phi^{\prime}\left((\phi b)^{n+1}(1)\right)=\frac{\phi^{\prime}(b 1)}{\phi^{\prime}\left(b(\phi b)^{n}(1)\right) \cdots \phi^{\prime}(b \phi b 1)}
$$

from one side and

$$
\phi^{\prime}\left((\phi b)^{n+1}(1)\right)=\frac{\phi^{\prime}\left((\phi b)^{n} 1\right)}{\phi^{\prime}\left(b(\phi b)^{n} 1\right)}=\cdots=\frac{\phi^{\prime}(1)}{\phi^{\prime}\left(b(\phi b)^{n} 1\right) \ldots \phi^{\prime}(b)}
$$

from the other side.

These quantities are equal since

$$
\phi^{\prime}(\phi b 1)=\frac{\phi^{\prime}(1)}{\phi^{\prime}(b)} \quad \text { i.e. } \quad \phi^{\prime}(-a)=\frac{1}{\phi^{\prime}(b)} .
$$

Hence $\phi$ is $C^{1}$ at junctions $(\phi b)^{n}(1)$ and, similarly, at junctions $(\phi b)^{n}(-1)$.

If $y$ is close to $\alpha$ then $y=(\phi b)^{2 n} x$ for large $n$ and some $x$. Hence

$$
\phi^{\prime}(y)=\phi^{\prime}\left((\phi b)^{2 n} x\right)=\frac{\phi^{\prime}(x)}{\phi^{\prime}\left(b(\phi b)^{2 n-1}(x)\right) \cdots \phi^{\prime}(b x)} .
$$

Since $\phi$ is $C^{1}$ on $[-a, 0]$ and $a \leq(\phi b)^{2} x \leq \phi(-a b)$, the denominator grows exponentially, so that $\phi^{\prime}(y) \rightarrow 0$ as $y \rightarrow \alpha_{+}$. Similarly, $\phi^{\prime}(y) \rightarrow 0$ as $y \rightarrow \beta_{-}=\alpha_{-}$. The proof is complete.

\section{Periodic repellors}

Suppose $\phi$ is a self-replicating map of $[-1,1]$ onto itself satisfying $\phi(-x)=-\phi(x)$, $\phi^{2}(b x)=b \phi(x)$ where $0<b<a<1$ and $\phi(-b)=a$. As we have noted, $\phi(-a)=b^{2}$ so that $\phi^{2}$ maps $[-a,-b]$ homeomorphically to an interval containing $[-a,-b]$. Hence there is at least one point $P_{2} \in(-a,-b)$ such that $\phi^{2}\left(P_{2}\right)=P_{2}$. We shall impose the hypothesis

$$
\phi^{2} \text { has only one fixed point in }[-a,-b]
$$

or

$$
\phi^{\prime}(x)>1 \text { in }(-a,-b)
$$


to ensure the uniqueness of $P_{2}$ in $(-a,-b)$. By symmetry we shall then also have $\phi^{2}\left(-P_{2}\right)=-P_{2}$ and hence $\phi\left(P_{2}\right)=-P_{2}$. Moreover, $P_{2}$ and $-P_{2}$ are repellors and, in fact, if $x \in(-a,-b)$ and $x \neq P_{2}$, then for some $N, \phi^{n}(x) \notin(-a,-b) \cup(b, a)$ for all $n \geq N$. We therefore have:

Lemma. Assuming (H.2), the non-wandering set of $\phi$ is disjoint from

$$
(-a,-b) \cup(b, a)-\left\{ \pm P_{2}\right\}
$$

\section{The non-wandering set}

Throughout this section we assume $\phi$ is a self-replicating Lorenz map satisfying (H.2).

We shall make use of the following (perhaps unecessarily strong) hypothesis:

The initial map (defined on $[-a, a]$ ) satisfies

$$
\frac{\phi(x)-\phi(y)}{x-y}<\frac{\lambda}{b}
$$

when $-b<y<x<0$, where $0<\lambda<1$.

THEOREM 3. If $\phi$ satisfies $\left(\mathrm{H}^{\prime \prime} .1\right)$ and (H.2), then the non-wandering set is a Cantor set, which is essentially the Morse minimal set, together with $2^{n}$ points of period $2^{n}$ for each $n=1,2, \ldots$.

Proof. It is convenient to consider the map $\psi:[0,1] \rightarrow[0,1]$ obtained from $\phi$ by factoring the symmetry $x \rightarrow-x . \psi$ then satisfies: $\psi^{2}(b x)=b \psi(x), \psi$ has only one fixed point in $(b, a)$ and $|(\psi(x)-\psi(y)) /(x-y)|<\lambda / b$ when $b a<y<x<b$, where $0<\lambda<1$. We also note that $\psi(0)=1, \psi(b)=a, \psi(a)=b^{2}, \psi(\alpha)=0$ and $\psi(1)=b$. Moreover, $\psi$ is decreasing on $[0, \alpha]$ and increasing on $[\alpha, 1] . \psi$ maps $[0, b]$ onto $[a, 1]$.

Let $W$ denote the wandering set together with periodic points. $W$ contains $(b, a)$ and, since $\psi$ is self-replicating, $W$ contains $b(b, a)=\left(b^{2}, b a\right)$ and therefore $\psi b(b, a)$ (figure 6). Repeating this argument $\boldsymbol{W}$ contains

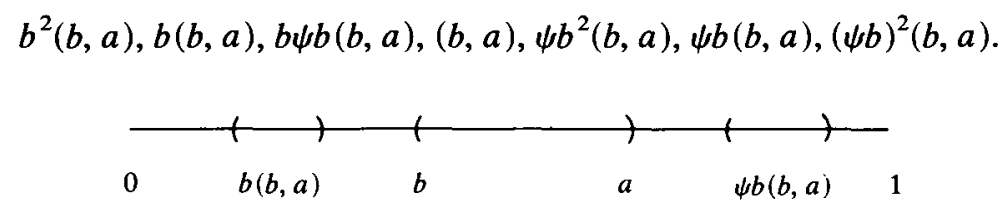

FIGURE 6

This process can be continued indefinitely so that $W$ contains the countable collection $\mathscr{C}$ of open intervals generated by applying the maps $b, \psi$ to the interval $(b, a)$ successively.

Suppose at any stage the maximum diameter of the complementary intervals is $d$. Then all neighbouring intervals in $\mathscr{C}$ are at most $d$ apart. Applying $b$ we then have that neighbouring $\mathscr{C}$ intervals in $[0, b]$ are at most $b d$ apart and, applying $\psi$, neighbouring $\mathscr{C}$ intervals in $[a, 1]$ are at most $\lambda d$ apart. Thus maximum diameters of complementary intervals decrease to zero with the process. In other words, the union of $\mathscr{C}$ intervals is everywhere dense, which shows that $W^{c}$ is nowhere dense. A simple 
compactness argument shows that $W^{c}$ is uncountable, i.e. $W^{c}$ is a Cantor set. From this we conclude that the non-wandering set $N$ of $\phi$ consists of the periodic points together with a Cantor set.

Let $I_{0}=N \cap[-1,0], I_{1}=N \cap[0,1]$. To each $x \in N$ we associate the sequence $x_{0}, x_{1}, \ldots\left(x_{i}=0,1\right)$ if $x \in \bigcap_{n=0}^{\infty} \phi^{-n} I_{x_{n}}$, and note that each $x$ has a unique sequence associated with it, unless some iteration of $x$ equals zero. Moreover, no two points of $N$ have the same sequence associated with them for this would mean an interval of points are non-wandering. Thus if we associate two sequences to zero and to all points which iterate to zero we can identify the non-wandering set with the corresponding set of sequences of zeros and ones.

Now $N$ contains the points of period $2,0101 \ldots$, and $1010 \ldots$ Using the self-replicating property of $\phi, N$ contains the point in

$$
\begin{aligned}
{[-b, b] } & \cap I_{0} \cap \phi^{-2}\left([-b, b] \cap I_{1}\right) \cap \phi^{-4}\left([-b, b] \cap I_{0}\right) \cap \cdots \\
& \subset\left(I_{0} \cap \phi^{-1} I_{1}\right) \cap \phi^{-2}\left(I_{1} \cap \phi^{-1} I_{0}\right) \cap \phi^{-4}\left(I_{0} \cap \phi^{-1} I_{1}\right) \cdots \\
& =01101001 \ldots
\end{aligned}
$$

Similarly, $N$ contains the point $10010110 \ldots$ In fact, $N$ contains the point $01100110 \ldots$ and its 4 -point periodic orbit. Again we can continue this line of reasoning indefinitely. It clearly amounts to the permissibility of making the substitutions 01 for 0 and 10 for 1 . At the next stage, for example, $N$ contains the point $0110100110010110 \ldots$ and its 8-point orbit.

Clearly, then, $N$ contains, for each $n \geq 1$, a periodic orbit of period $2^{n}$.

Consider the Morse sequences (cf. [6], [7]) obtained by the following process:

$$
01,0110,01101001, \ldots, s \text {. }
$$

It is not difficult to see that the compact set obtained as the limit set of $s$ together with its shift iterations is exactly the same as the limit of the set of periodic sequences obtained above. We shall call this limit set (together with the shift) the Morse minimal set. (Strictly speaking the Morse minimal set, as it appears in the literature, is obtained from a two-sided infinite sequence.)

Thus $N$ contains, together with the above periodic points, the Morse minimal set. (Note, however, that the zero point is associated with the Morse sequence and its reflection $10010110 \ldots$. .

Finally, we contend that $N$ contains no other points. This can best be seen by noting that, for every $\varepsilon>0, N$ is contained in a finite union of closed intervals of diameter less than $\varepsilon$, and in each of these intervals there is a periodic point. Thus the periodic points are dense in $N$.

\section{The map $\psi$}

We conclude with a brief description of the map $\psi$. Evidently, if we specify a strictly decreasing continuous map $\psi$ of $[0, a]$ into $[0,1]$ with the property that $\psi(0)=1$, $\psi(a)=b^{2}, \psi(b)=a(0<b<a<1)$, then $\psi$ can be extended uniquely to a continuous map $\psi:[0,1] \rightarrow[0,1]$ which is strictly decreasing to some point $\beta$ and strictly 
increasing in $[\alpha, 1]$ (where $\beta \leq \alpha$ and $\psi$ is zero on $[\beta, \alpha], a<\beta<\alpha<1$ ) and which is self-replicating $\left(\psi^{2}(b x)=b \psi(x)\right)$. If the initial map $\psi$ has only one fixed point in $[b, a]$, and $\psi b$ has no fixed points in $[a, 1]$, then the fixed point is a repellor which renders the points of $(b, a)$ wandering (except for the fixed point) and $\psi$ is zero at only one point $(\alpha=\beta)$.

If in addition $\psi$ is $C^{1}$ and $\psi^{\prime}(a) \psi^{\prime}(b)=1$, then the extended $\psi$ is also $C^{1}$ (even at $\alpha$, since $\psi^{\prime}(\alpha)=0$.)

If we assume $\left|\psi^{\prime}(x)\right|<\lambda / b$ (in $(0, b)$ ) where $0<\lambda<1$, then the non-wandering set of the extended $\psi$ consists of a Cantor set together with $2^{n}$ periodic points of period $2^{n}$ for $n=0,1, \ldots$

The restriction of $\psi$ to the Cantor set is precisely the so-called adding machine, i.e. the 2 -adic integers together with the transformation which adds $(1,0,0, \ldots)$ to each point.

This last result is achieved by factoring the Morse minimal set by the symmetry $x \rightarrow x^{\prime}$ where $x^{\prime}$ is obtained from the sequence $x$ by interchanging zeros and ones (cf. [6], [7]). However, it is worth remarking that the result may be obtained directly from our representation of the Morse minimal set and the properties of $\phi$ and $\psi$.

\section{Characteristic exponents}

We shall omit the general theory of characteristic exponents and simply define these quantities for piecewise $C^{1}$ maps of an interval preserving an ergodic probability measure.

Let $f$ be a piecewise $C^{1}$ map preserving the ergodic probability $\mu$. Then

$$
\frac{1}{n} \log \left|\left(f^{n}\right)^{\prime}(x)\right|=\frac{1}{n} \sum_{i=0}^{n-1} \log \left|f^{\prime}\left(f^{i}(x)\right)\right| \rightarrow \int \log \left|f^{\prime}(x)\right| d \mu
$$

for almost all $x[\mu]$.

We denote this limit by $\chi_{\mu}$. It is known as the characteristic exponent of $f$ with respect to $\mu$.

It is clear that, if $\phi$ is a self-replicating Lorenz map satisfying $\left(\mathrm{H}^{\prime} .2\right),\left(\mathrm{H}^{\prime \prime} .1\right)$ and $\phi^{\prime}(a) \phi^{\prime}(b)=1$, then it preserves only the following ergodic measures: $m_{1}, m_{2}, \ldots, m$, where $m_{n}$ is the atomic measure assigning $1 / 2^{n}$ to each point of the periodic orbit of period $2^{n}$, and $m$ is the unique invariant probability on $M$ obtainable as the weak* limit of the measures $m_{n}$.

We shall compute $\chi_{n}=\chi_{m_{n}}, n=1,2, \ldots, \chi_{\infty}=\chi_{m}$. Let $P_{2}$ be the point of period 2 in $(-a,-b)$, then $\phi^{\prime}\left(P_{2}\right)>1$ and

$$
\chi_{1}=\frac{1}{2}\left[\log \phi^{\prime}\left(P_{2}\right)+\log \phi^{\prime}\left(\phi P_{2}\right)\right]=\log \phi^{\prime}\left(P_{2}\right)>0 .
$$

Evidently, $b^{n} P_{2}=P_{2^{n+1}}$ is a point of period $2^{n+1}(n=0,1, \ldots)$, since $\phi^{2^{n}}\left(b^{n} x\right)=$ $b^{n} \phi(x)$ and hence $\phi^{2^{n+1}}\left(b^{n} P_{2}\right)=\phi^{2 n} \phi^{2^{n}}\left(b^{n} P_{2}\right)=\phi^{2 n} b^{n} \phi P_{2}=b^{n} \phi^{2} P_{2}=b^{n} P_{2}$.

Thus $\left[\phi^{2 n}\right]^{\prime}\left(b^{n} x\right)=\phi^{\prime}(x)$ and

$$
\chi_{n+1}=\frac{1}{2^{n}} \log \left[\phi^{2^{n}}\right]^{\prime}\left(b^{n} P_{2}\right)=\frac{1}{2^{n}} \log \phi^{\prime}\left(P_{2}\right)=\frac{1}{2^{n}} \chi_{1} .
$$

Finally, we show that $\chi_{\infty}=0$. (We cannot justify the equation $\lim _{n \rightarrow \infty} \chi_{n}=\chi_{\infty}$ directly, as $\log \phi^{\prime}(x)$ is not continuous since $\phi^{\prime}(\alpha)=0$.) We shall need the fact that $\phi$ is 
essentially one-one with respect to $m$, a fact easily deducible from properties of the Morse minimal set or from properties of the 2-adic adding machine.

Since $\phi^{2} b x=b \phi x$ we have $\phi^{2} b E=b \phi E$ for all Borel sets $E$, and hence

$$
m \phi^{2} b E=m b E=m b \phi E .
$$

Thus $m b$ is a $\phi$ invariant measure. By the uniqueness of $m$ this means that $m b E=K \cdot m E$ for some constant $K$ and all Borel sets $E$. Hence $m[-b, b]=K$. However, $\phi[-b, b]=[-1,-a] \cup[a, 1]$, which is disjoint from $[-b, b]$, and $\phi^{2}[-b, b]=[-a, a]=[-b, b] \cup$ (null set). Therefore $m[-b, b]=\frac{1}{2}$ (i.e. $K=\frac{1}{2}$ ). In other words $m b E=\frac{1}{2} m E$ for all Borel sets $E$.

$$
\text { Let } \begin{aligned}
& I_{0}=\int_{-a}^{0} \log \phi^{\prime}(x) d m, \\
& \qquad \begin{aligned}
I_{n} & =\int_{(\phi b)^{n}[-a, 0]} \log \phi^{\prime}(x) d m \\
& =\int_{(\phi b)^{n+1}(1)}^{(\phi b)^{n-1}(1)} \log \phi^{\prime}(x) d m, \quad n=1,2, \ldots .
\end{aligned}
\end{aligned}
$$

Using the fact that $m b E=\frac{1}{2} m E$ for all Borel sets $E$, we have

By (3.1) we know that

$$
I_{n}=\frac{1}{2^{n}} \int_{-a}^{0} \log \phi^{\prime}\left((\phi b)^{n} x\right) d m
$$

$$
\phi^{\prime}\left((\phi b)^{n} x\right)=\frac{\phi^{\prime}(x)}{\phi^{\prime}\left(b(\phi b)^{n-1} x\right) \cdots \phi^{\prime}(b x)}
$$

and hence

$$
I_{n}=\frac{1}{2^{n}}\left(\int_{-a}^{0} \log \phi^{\prime}(x) d m-\sum_{i=0}^{n-1} \int_{-a}^{0} \log \phi^{\prime}\left(b(\phi b)^{i} x\right) d m\right) .
$$

Consequently,

$$
\begin{aligned}
\sum_{n=0}^{\infty} I_{n} & =I_{0}+\sum_{n=1}^{\infty} \frac{1}{2^{n}}\left[I_{0}-\int_{-a}^{0} \sum_{i=0}^{n-1} \log \phi^{\prime}\left(b(\phi b)^{i} x\right) d m\right] \\
& =2 I_{0}=\sum_{n=0}^{\infty} \int_{-a}^{0} \frac{1}{2^{n}} \log \phi^{\prime}\left(b(\phi b)^{n} x\right) d m \\
& =2 I_{0}-\int_{-a}^{0} \log \phi^{\prime}(b x) d m-\int_{(\phi b)^{2}(1)}^{1} \log \phi^{\prime}(b x) d m-\int_{(\phi b)^{3}(1)}^{(\phi b)(1)} \log \phi^{\prime}(b x) d m-\cdots \\
& =2 I_{0}-\int_{-a}^{0} \log \phi^{\prime}(b x) d m-\int_{-\alpha}^{-a} \log \phi^{\prime}(b x) d m-\int_{\alpha}^{1} \log \phi^{\prime}(b x) d m \\
& =2 I_{0}-\int_{-a}^{0} \log \phi^{\prime}(b x) d m-\int_{-1}^{-a} \log \phi^{\prime}(b x) d m \\
& =2 I_{0}-\int_{-1}^{0} \log \phi^{\prime}(b x) d m \\
& =2 I_{0}-2 \int_{-b}^{0} \log \phi^{\prime}(x) d m=0 .
\end{aligned}
$$


Hence

$$
\chi_{\infty}=2 \sum_{n=0}^{\infty} I_{n}=0
$$

\section{REFERENCES}

[1] P. Collet, J.-P. Eckmann \& H. Kock. Period doubling bifurcations for families of maps on $\mathbb{R}^{n}$. (To appear.)

[2] M. J. Feigenbaum. Quantitative universality for a class of non-linear transformations. J. Stat. Physics 19 (1978), 25-52.

[3] J. Guckenheimer. A strange, strange attractor. In The Hopf Bifurcation (ed. Marsden \& McCracken) Appl. Math. Sci. Springer-Verlag: Berlin, 1976.

[4] F. Hofbauer. The maximal measure for the transformation $T: x \rightarrow \beta x+\alpha \bmod 1$. Journal L.M.S. (in the press).

[5] L. Jonker and D. Rand. Bifurcations in one dimension I: The non-wandering set. Invent. math. 62 (1981), 347-365.

[6] S. Kakutani. Ergodic theory of shift transformations. Proc. Berkeley Symp. Math. Stat. 5. Prob. 11 (1967), 405-414.

[7] M. Keane. Generalised Morse sequence. Z. Wahrscheinlichkeitstheorie verw. Geb. 10 (1968), $335-353$

[8] J. Milnor \& W. Thurston. On iterated maps of the interval I and II. Princeton University and the Institute for Advanced Studies, Princeton preprints.

[9] M. Misiurewicz. Structure of mappings of an interval with entropy. (To appear.)

[10] M. R. Palmer. Mixing properties of linear mod 1 maps of the unit interval. (To appear.)

[11] W. Parry. The Lorenz attractor and a related population model. Proceedings of Ergodic Theory conference. Oberwolfach 1978. Lecture Notes in Math. no. 729. Springer-Verlag: Berlin, 1978.

[12] R. F. Williams. The structure of Lorenz attractors. Publ. Math. I.H.E.S. 50 (1979) 321-347. 